\title{
RELATIVITY EFFECTS IN THE ROTATION OF THE EARTH AND THE MOTION OF ATMOSPHERIC MASSES
}

\author{
V.G. Shkodrov and V.G. Ivanova \\ Department of astronomy and National Astronomical Observatory \\ at the Bulgarian Academy of Sciences, \\ Sofia, Bulgaria
}

\begin{abstract}
On the basis of observational data on atmospheric pressure (1963-1967), the variation of the moment of inertia, and, with certain restrictions, the changes in the angular velocity of the Earth are obtained. The numerical results derived are compared to the relativity effects in Earth rotation. The comparison shows that both effects are equal in periods and very close in amplitudes.
\end{abstract}

So far, dynamical theories have not been sufficiently accurate to explain in details the variations of the Earth's rotation rate. Variations of Earth's angular velocity have been explained only partially, usually taking into account only some of the possible physical causes. This is due mainly to the very great complexity of the problem under discussion.

We do not claim that we propose here a dynamical theory that takes into account all the many causes of the annual variations of the Earth's rate of rotation. Our purpose is much more modest. This paper is making an attempt to point out that the amplitudes of these variations, due to the changes of the moment of inertia of the atmosphere and the rotation effect of Einstein's theory of general relativity are of the same order of magnitude. This means that any future theory must include the relativity effect. The atmospheric momentum of inertia $\mathrm{C}_{\mathrm{a}}$ has been calculated in our paper (Ivanova, 1980). The calculations were performed using the method proposed by Shkodrov (1979 and 1980) on the basis of data on atmospheric pressure upon the Earth's surface, kindly supplied by the Hydrometeorological Centre of the Soviet Academy of Sciences. The results of the calculations for the period 1963-1967 are given in Fig. 1. As it can be seen from Fig. 1, the variations of the momentum of inertia $C_{a}$ have a seasonal aspect.

On the other hand, as it is well known, the orbital motion and the angular momentum for the rotation are not independent. In the case of a Newtonian theory of motion, the interrelation of the two momenta is due to the non-spheric symmetry in the mass distribution in the body. With the Einstein's theory of general relativity such an interrelation exists also in the case of spherical symmetry. To determine the influence of the orbital motion on the rotation rate $\delta \omega_{r}$, we shall use the equation 289

J. Kovalevsky and V. A. Brumberg (eds.), Relativity in Celestial Mechanics and Astrometry, 289-292.

(C) 1986 by the IAU. 


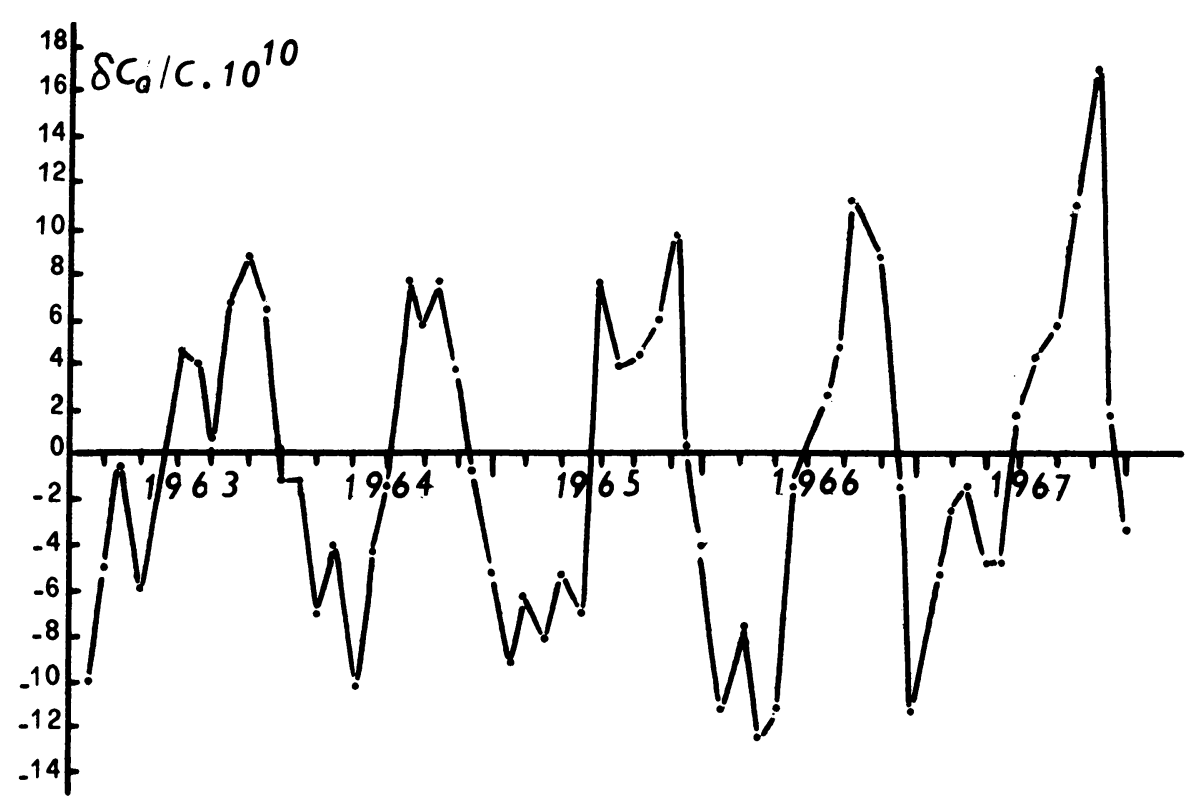

Figure 1. Seasonal variations of the moment of inertia of the atmosphere $\mathrm{C}_{\mathrm{a}}$

obtained by Brumberg (1970):

$$
\delta \omega_{r} / \omega^{\prime}=5 \mathrm{Gm}_{\mathrm{O}} \mathrm{ec}^{-2} \mathrm{a}^{-1}\left(1-\mathrm{e}^{2}\right)^{-1} \cos \mathrm{f}
$$

where $G$ is the gravitational constant, $c$ is the velocity flight, a is the semi-major axis of the planetary orbit, e, its eccentricity and $f$, the true anomaly. From the above equation we obtain for the Earth :

$$
\delta \omega_{r} / \omega^{\prime}=-0,85 \cdot 10^{-9} \cos \mathrm{f}
$$

In order to compare our results shown in Fig. 1 to the effect of rotation (2), we assume that the law of conservation of the angular momentum takes also into account the relativity correction (2) :

$$
\mathrm{C} \omega^{\prime}=\text { const } \text {. }
$$

where $\omega^{\prime}=\omega+\omega_{r}$. This means that we have a dynamic system, taking into account the influence (2) of orbital motion on Earth's rotation rate. Another assumption we made is that $\omega^{\prime}$ is a directly observed value. In this case we obtain from (3)

$$
\delta \mathrm{C} / \mathrm{C}+\delta \omega / \omega^{\prime}+\delta \omega_{\mathrm{r}} / \omega^{\prime}=0
$$

Assuming that the variations of $C$ and $\omega$ are caused only by the change of distribution of the atmospheric masses $\left(C=C_{a}, \omega=\omega_{a}\right)$, we 
obtain :

$$
-\delta \omega_{a} / \omega^{\prime}=\delta C_{a} / C+\delta \omega_{r} / \omega^{\prime}
$$

Consequently, variations $\delta \omega_{a}$ are to be estimated from the addition of the curve shown in Fig.1 and the one obtained from (2). On Fig. 2, variations $\delta \omega_{a} / \omega^{\prime}, \delta c_{a} / C$ and $\delta \omega_{r} / \omega^{\prime}$ are shown. Note that in this figure, $\delta \mathrm{C}_{\mathrm{a}} / \mathrm{C}$ is the mean annual variation, estimated from the mean value of the variations of the momentum of inertia $C_{a}$ given in Fig. 1. As it results from Fig. 2, $\delta c_{a} / C$ and $\delta \omega_{r} / \omega^{\prime}$ are not only of the same order of magnitude but they have approximately the same amplitudes, the phase between the two curves being approximately $40^{\circ}$ (or 1.3 month).

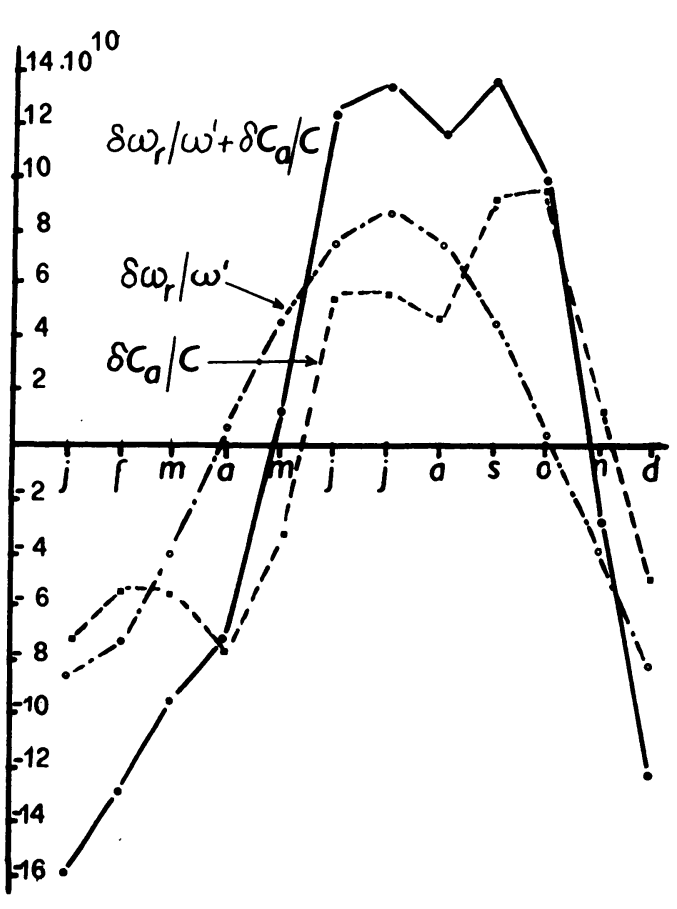

Figure 2. Seasona1 variations of angular velocity $\delta \omega_{\mathrm{a}} / \omega^{\prime}$ of the momentum of inertia of the atmosphere $\delta \mathrm{C}_{\mathrm{a}} / \mathrm{C}$ and the relativity correction $\delta \omega_{\mathrm{r}} / \omega^{\prime}$.

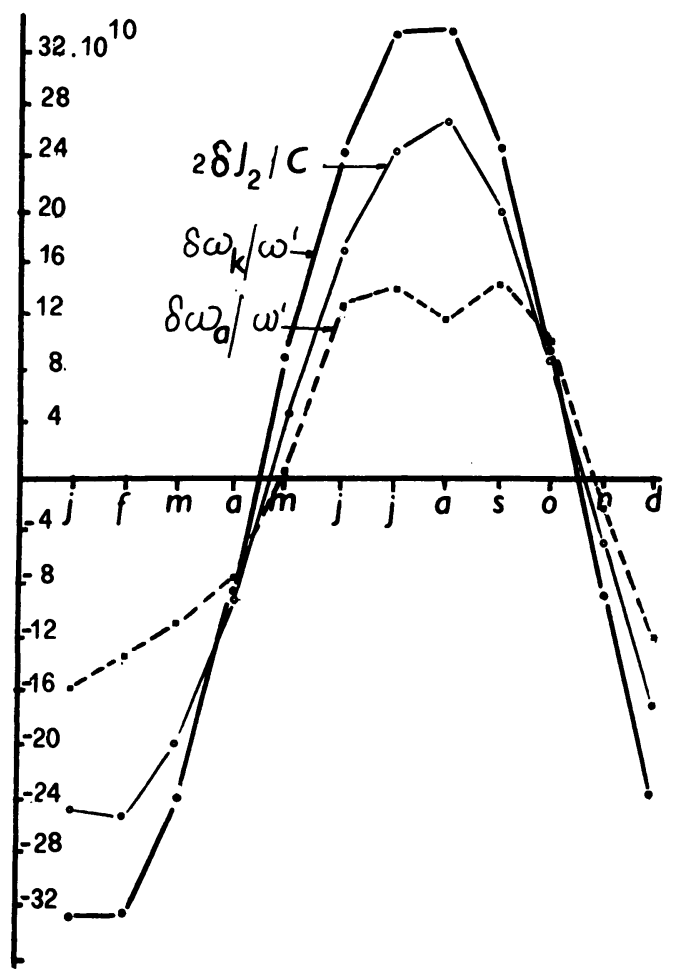

Figure 3. Seasonal variations of angular velocity $\delta \omega_{k} / \omega^{\prime}$ obtained by Kozai plus the relativity correction. shown.

In the same Fig. 2, the variation of $\delta \omega_{a} / \omega^{\prime}$, obtained from (5), is Applying (4) to the results obtained by Kozai (1970), it follows :

$$
-\delta \omega_{k} / \omega^{\prime}=2 \delta J_{2}+\delta \omega_{r} / \omega^{\prime}
$$


where $\delta \omega_{k} / \omega^{\prime}$ is the variation of the Earth's angular velocity obtained by Kozai, plus the relativity correction, and $\delta \mathrm{J}_{2}$ is the variation of th second zonal harmonic of the geopotential,obtained from the analysis of the motion of artificial satellites. Figure 3 gives the variations of $\delta \omega_{k} / \omega^{\prime}$. One can also see, on the same figure, the curve obtained by Kozai without the relativity correction.

In conclusion, the results shown here and their brief analysis suggest that any dynamic theory claiming to explain accurately the seasonal variations of the Earth's rate of rotation, would be fundamentally irrelevant and incompatible with observations if it fails to consider the rela. tivity effect. The explanation of seasonal variations taking into accoun 1 only the atmospheric effects is hardly acceptable from a physical point of view. In a certain sense this is coroborated by the comparison of $\delta \omega_{k} / \omega^{\prime}$ and $\delta \omega_{\mathrm{a}} / \omega^{\prime}$ on Fig.3. Most probably, the larger amplitude of $\delta \omega_{k} / \omega^{\prime}$ obtained by Kozai is due to the fact that his results include all the effects produced by the mass redistribution within the interior of the Earth. In any case, tentatives to explain the variations of the Earth's rotation only using atmospheric processes are rather dangerous because both effects considered here are of the same order of magnitude and, frequently, can hardly be separated.

\section{REFERENCES}

Brumberg, A., 1970, Bu11. Institut. Theor. Astr. USSR, 12 $=$, 6, 441. Ivanova, V.G., 1980, Compt. Rend. Acad. Bulg. Sci., 33 Kozai, Y, 1970, SAO Special Report ${ }^{\circ} 312$.

Shkodrov, V.G. and Ivanova, V.G., 1979, Compt. Rend. Acad. Bug. Sci., $\underline{3} 2,12,1631$

Shkodrov, V.G. and Ivanova, V.G., 1980, Bulg. Geophys. J., $\underline{\underline{6}, 4,20 .}$ 